Discussion Paper No. 774

\title{
EFFICIENCY VERSUS ECONOMY OF TIME \\ IN MULTI-UNIT DESCENDING AUCTION: \\ THE ROLE OF "MARI" AT FLOWER MARKETS \\ IN JAPAN
}

\author{
Minoru Kitahara \\ Ryo Ogawa
}

April 2010

The Institute of Social and Economic Research

Osaka University

6-1 Mihogaoka, Ibaraki, Osaka 567-0047, Japan 


\title{
Efficiency versus Economy of Time in
}

\section{Multi-Unit Descending Auction:}

\section{The Role of "Mari" at Flower Markets in}

\author{
Japan*
}

\author{
Minoru Kitahara ${ }^{\dagger}$ \\ and \\ Ryo Ogawa ${ }^{\ddagger}$
}

First Draft: February 2006

This Version: February 2010

\footnotetext{
${ }^{*}$ The authors are grateful to Michihiro Kandori, Toshihiro Matsumura, Kuniyoshi Saito, Kiri Sakahara, Daisuke Shimizu, and Takahiro Watanabe for comments and discussions. The authors are also grateful to seminar/conference participants at the 2006 Autumn Meeting of the Japanese Economic Association, the 2006 South and South-east Asia Meeting of the Econometric Society in Chennai, and the 2008 Far Eastern Meeting of the Econometric Society in Singapore. Financial supports from Japan Society for the Promotion of Science are gratefully acknowledged.

${ }^{\dagger}$ JSPS Research Fellow, Graduate School of Social Sciences, Tokyo Metropolitan University, 1-1 Minami-Osawa, Hachioji-shi, Tokyo 192-0397, Japan, and Visiting Research Fellow, Advanced Research Institute for the Sciences and Humanities, Nihon University, 12-5 Goban-cho, Chiyoda-ku, Tokyo 102-8251, Japan (mkitahar@tmu. ac. jp).

${ }^{\ddagger}$ Corresponding author. Institute of Social and Economic Research, Osaka University, 6-1 Mihogaoka, Ibaraki, Osaka 567-0047, Japan (r-ogawa@iser .osaka-u. ac. jp).
} 


\begin{abstract}
The auctioning rule in Japanese flower markets is a slightly modified version of that of the original Dutch flower auction. At Japanese flower markets, there is an additional stage, called "mari", where buyers who lost in the previous auction can apply for purchasing the remainder of flowers at the same price as in the previous auction. We investigate the role of "mari" in multi-unit descending auction, and show that "mari" extensively speeds up the market procedure at the cost of sufficiently small loss of efficiency, compared to the original Dutch sequential auction.
\end{abstract}

\title{
1 Introduction
}

Descending price auction (or, Dutch auction) is widely used at wholesale flower markets in countries such as the Netherlands and Japan among others. The Dutch market is leading in the worldwide market share, and Ota Floriculture Auction in Tokyo is the second largest ${ }^{1}$. The Dutch style of the flower auction originates in the Netherlands (as is clear from the name) and was imported into Japan, but in the process of adoption of the descending style, many of flower markets in Japan, including Ota, made a slight modification to the original rule. In this paper we call the modified rule in Japan as mari, which comes from marketmen's jargon referring to the modification. This paper investigates both advantages and disadvantages of mari, and shows that mari extensively speeds up the market procedure at the cost of sufficiently small loss of efficiency, compared to the original

\footnotetext{
${ }^{1}$ Two organizations of flower auctions, Flora Holland and Aalsmeer, were competing in the Netherlands until 2007. In 2008, Flora Holland and Aalsmeer merged, and then Ota Floriculture in Tokyo became the second largest.
} 
Dutch sequential auction. $^{2}$

The key instrument at those markets is the "auction clock", beginning with a high price which is then gradually lowered. If a buyer desires to buy the good at the price on the clock, he pushes the button then the clock stops. The good is won by the buyer who was the first to stop the clock, and the winner pays the price displayed on the clock to the auctioneer.

When several identical goods are for sale, some options are open to the seller, even when the market sticks to the descending price style as the basic procedure. For instance, the seller must decide how to sell the remainder of the goods after some buyer has won one good. The remainder is brought up to the auction again at the Ducth flower auctions, ${ }^{3}$ whereas at many wholesale flower markets in Japan, the remainder is sold by the peculiar method called mari.

Roughly speaking, mari allows other buyers who did not win the good at the previous auction to apply for purchasing the remainder (if any) at the same price as the winner in the previous auction paid. If the number of applicants is less than the number of the remainder then all the applications are accepted, while the remainder is randomly allocated between applicants if the number of applicants exceeds the number of the remainder.

This paper investigates the effect of mari to the equilibrium outcome of the auction. One interesting nature would be that when the number of applicants exceeds the number of the remainder, the random allocation in mari may cause inefficiency: that is, some portion of the remaining goods

\footnotetext{
${ }^{2}$ In an interview at the Ota Floriculture Auction, a director who organizes the information system of the auctioning room pointed out that they acutally have a "feeling" that mari contributes to the economy of time at the market.

${ }^{3}$ Such situations in which the goods are sold sequentially are called "sequential auctions" in the literature. The analysis of sequential auctions originates with Milgrom and Weber [3] and Weber [4].
} 
may be allocated to the buyer whose value is lower among the applicants. On the other hand, mari may contribute to the economy of time in the sense that it decreases the number of auctions that should be held until all of the remainder be sold out. The purpose of the paper is to show that addition of mari to the sequential descending auction extensively reduces the number of auctions to be held, while the loss of efficiency is sufficiently small.

In investigating the relationship between efficiency and economy of time, there can be various ways of evaluating the trade-off. In this paper, we define two measures, "efficiency loss rate" $(L R)$ and "round reduction rate" $(R R)$, and examines the asymtotic property of $L R$ and $R R$ when the numbers of buyers and goods increase to infinity. $L R$ is defined as the ratio of the expected welfare loss in mari to the expected welfare if the goods were to be allocated efficiently. If such $L R$ is closer to zero, we see that the loss of efficiency is smaller. $R R$, on the other hand, is defined as the ratio of the expected number of goods sold in mari to the number of the remainder. We regard that the mari contributes more to the economy of time when $R R$ is closer to one. Our result actually shows that $L R$ converges to 0 while $R R$ converges to 1 when the number of buyers and that of goods increase to infinity (Theorem in page 19).

The rest of the paper is organised as follows. We formulate the sequential descending auction with mari in Section 2. In Section 3, we investigate the symmetric equilibrium of the game and provide some characteristics. Main result on the trade-off between efficiency and economy of time is presented in Section 4, in which we argue that mari extensively reduces the number of auctions while the loss of efficiency is sufficiently small. Section 5 provides concluding remarks. 


\section{The Model}

In this section, we introduce the sequential descending auction model with mari.

To formulate the problem, suppose that there are $k$ identical goods for sale and $N$ buyers. We assume that each buyer can buy only one item; that is, we limit our attention to the unit-demand case. The goods are to be sold in the following procedure:

1. The "auction clock" starts from a sufficiently high price and goes down until one of the buyers stops it.

2. The buyer who stopped the clock wins the good at the price on the clock.

3. Other buyers can apply to purchasing the remaining goods at the same price being paid by the winner in step 2 (mari).

(a) If the number of buyers who applied to the purchase is greater than the number of remaining goods (that is, $k-1$ ), then the goods are allocated randomly between the applying buyers. Put another way, if the number of applying buyers is $j \geq k-1$, then each buyer wins the good with (ex ante) probability $j /(k-1)$.

(b) Otherwise, all the applying buyers can win the good.

4. The remaining goods will be sold in the usual sequential descending auction without mari. ${ }^{4}$

\footnotetext{
${ }^{4}$ In the real Japanese flower auctions, mari may happen for several times. That is, after mari (step 3), the auctioning procedure simply goes back to step 1 . The existence of mari in and after the second round may affect how mari in the first round works. In order to avoid such effects, in this paper we focus on a simplified game in which mari can come up only in the first round.
} 
We let $t_{i}$ denote $i$ th buyer's type (value), and assume that the types are independently drawn from the uniform distribution on [0,1]. Each buyer attains the payoff of $t_{i}-p$ if the buyer wins the good and the winning price is $p$, and the payoff of 0 if the buyer fails to win the good. We assume that the buyers are risk-neutral.

The strategy of the buyers comprises following components. One is the decision about on what price the buyer stops the "auction clock" in the first round (depending on her type $t$ ), another is the decision about whether to apply in mari (depending on the price $p$ in the first round and on her type $t$ ), and the other is how to act in the sequential auction for the remaining goods if she either does not apply or loses in mari (depending on the price $p$, her type $t$, and the numbers of remaining buyers and remaining goods).

In this paper we focus on the symmetric equilibrium as in the following form: there exist $\beta_{N, k}$ and $\mu_{N, k}$ such that (i) type-t buyer stops the clock at $\beta_{N, k} t$ in the first round, (ii) if the clock stopped at the price $p=\beta_{N, k} \bar{t}$ in the first round, then other buyers participate in mari if and only if the type $t$ is higher than $\mu_{N, k} \bar{t}$, and (iii) the buyers follow symmetric equilibrium strategies in the sequential descending auction in 4 .

\section{Equilibrium Strategy}

In this section, we investigate the equilibrium strategy of buyers in the auction with mari. We also provide some equations characterizing $\mu_{N, k}$ and $\beta_{N, k}$ in the equilibrium, which will be extensively used in exlopring analysis of the trade-off between efficiency and economy of time in the next section.

In the beginning, we should obtain the symmetric equilibrium strategy in the auctions after mari when there is still remainder of the goods. It 
is easily shown that the bidding strategy in the equilibrium after mari is identical to that in the equilibrium of the usual sequential auction without mari, which is provided as

$$
b_{M, \ell}(t)=\frac{M-\ell}{M} t
$$

where $M$ is the number of remaining buyers and $\ell$ is that of remaining goods. ${ }^{5}$

Given the equilibrium strategy in the auctions after mari, $b_{M, \ell}(t)$, we investigate the buyers' decision about whether to apply in mari, for arbitrarily fixed bidding strategy in the first round, $\beta$. Suppose that type- $\bar{t}$ buyer won the good in the first round, that is, the winner bid $\beta \bar{t}$ and won the good with payment $\beta \bar{t}$. Then, in mari, each of remaining $N-1$ buyers, whose types are independently distributed uniformly on $[0, \bar{t})$, has a chance to apply for purchasing the good at the price $\beta \bar{t}$.

As was noted in the previous section, we are now interested in the linear symmetric equilibrium in which each buyer apply in mari if and only if the buyer's type is no less than $\mu \bar{t}$. To investigate the symmetric equilibrium, in the following, we study one typical buyer's decision whether to apply in mari, given other $N-2$ buyers' strategy $\mu$.

If the buyer decides to apply in mari, the probability of winning the good depends on the number of other applicants, $j$. If $j<k-1$, the buyer will be able to win the good with probability one, whereas the buyer wins with probability $(k-1) /(j+1)$ if $j \geq k-1$. As each of other buyers apply in mari with probability of $1-\mu$ ex ante, the probability that the number of other applicants equals to $j$ is $\left(\begin{array}{c}N-2 \\ j\end{array}\right)(1-\mu)^{j} \mu^{N-2-j}$, and therefore the probability

\footnotetext{
${ }^{5}$ See Milgrom and Weber [3] and Weber [4].
} 
that the buyer wins the good can be written as

$$
\sum_{i=0}^{k-2}\left(\begin{array}{c}
N-2 \\
i
\end{array}\right)(1-\mu)^{i} \mu^{N-2-i}+\sum_{i=k-1}^{N-2}\left(\begin{array}{c}
N-2 \\
i
\end{array}\right)(1-\mu)^{i} \mu^{N-2-i} \frac{k-1}{i+1}
$$

In the economy of notation, we write the expression as

$$
\mathbb{E}_{N-2}^{1-\mu}\left[\mathbf{1}_{j<k-1}+\frac{k-1}{j+1} \mathbf{1}_{j \geq k-1}\right]
$$

in the following, where the function $\mathbb{E}_{m}^{1-\mu}[f(j)]$ is defined as ${ }^{6}$

$$
\mathbb{E}_{m}^{1-\mu}[f(j)] \equiv \sum_{i=0}^{m}\left(\begin{array}{c}
m \\
i
\end{array}\right)(1-\mu)^{i} \mu^{m-i} f(i),
$$

and $\mathbf{1}$ is the indicator function.

To summarize, if the other buyers are following the mari participation strategy $\mu$, then the expected payoff of the buyer with type $t$ who decides to apply in mari, denoted by $\pi_{m}(t)$ would be

$$
\pi_{m}(t)=(t-\beta \bar{t}) \mathbb{E}_{N-2}^{1-\mu}\left[\mathbf{1}_{j<k-1}+\frac{k-1}{j+1} \mathbf{1}_{j \geq k-1}\right]
$$

where $t-\beta \bar{t}$ is the ex post payoff when the buyer successfully wins the good in mari and the $\mathbb{E}$ thing represents the probability that the buyer wins the good in mari.

If the buyer does not apply in mari, on the other hand, the expected

\footnotetext{
${ }^{6} \mathbb{E}_{m}^{1-\mu}[f(j)]$ can be understood as the expectation of function $f(j)$ when $j$ follows the binomial distribution with parameters $1-\mu$ and $m$.
} 
payoff, denoted by $\pi_{w}(t)$, can be written as

$$
\pi_{w}(t)= \begin{cases}\mathbb{E}_{N-2}^{1-\mu}\left[\left(t-b_{N-1-j, k-1-j}(\mu \bar{t})\right) \mathbf{1}_{j<k-1}\right] & \text { if } t \geq \mu \bar{t} \\ \mathbb{E}_{N-2}^{1-\mu}\left[\left(\int_{0}^{t} x_{N-1-j, k-1-j}\left(\frac{s}{\mu \bar{t}}\right) d s\right) \mathbf{1}_{j<k-1}\right] & \text { if } t<\mu \bar{t}\end{cases}
$$

We shall explain the two expressions in due order. For case of $t \geq \mu \bar{t}$, the buyer's optimal action in the auctions after mari (if any) is to act as if type- $\mu \bar{t}$ buyer in the equilibrium. This is because there are no other buyers with types more than $\mu \bar{t}$ in the auctions after mari as long as other buyers are following $\mu$. Moreover, this buyer will win with probability one in the auction just after mari (if any). Hence, the expected payoff when this buyer does not participate in mari can be written as in (4), where $b_{M, \ell}(t)$ is the equilibrium strategy in the auctions after mari as given in (1).

For case of $t<\mu \bar{t}$, we define $x_{M, \ell}(s)$ is as

$$
x_{M, \ell}(s) \equiv \sum_{i=0}^{\ell-1}\left(\begin{array}{c}
M-1 \\
i
\end{array}\right)(1-s)^{i} s^{M-1-i}
$$

That is, $x_{M, \ell}(s)$ denotes the probability that type-s buyer is at least $\ell$-highest among $M$ buyers whose types are independently drawn from the uniform distribution over $[0,1] .^{7}$

Here we have the following characterization of $\mu$ in the equilibrium in mari stage.

\footnotetext{
${ }^{7}$ Conceptually, the expected payoff from deferring participation in mari is written in more complex form than the one given in (4). In calculating the expected payoff, we must take into consideration all of the possibilities that the buyer wins the first after mari, the buyer loses the first but wins the second after mari, and so forth. Given the symmetric equilibrium strategy in the usual sequential descending auction following mari, which is already given in (1), we can rewrite the (complex) expected payoff as the simple form given in (4).
} 
Proposition 1. Suppose that the buyers are taking first-round bidding strategy $\beta$ and type- $\bar{t}$ buyer won the good in the first round. Then there exists unique linear symmetric equilibrium in mari stage in which each of the remaining buyers participates in mari if and only if the buyer's type is no less than $\mu \bar{t}$, where $\mu$ is given as follows.

1. If $\beta \geq(N-k) /(N-1)$, then $\mu=1$.

2. If $0<\beta<(N-k) /(N-1)$, then $\mu$ is the unique solution of

$$
(1-\beta) \mathbb{E}_{N-2}^{1-\mu}\left[\mathbf{1}_{j<k-1}+\frac{k-1}{j+1} \mathbf{1}_{j \geq k-1}\right]=\frac{k-1}{N-1} .
$$

Proof. (Necessity). If $\mu$ constitutes an equilibrium when $0<\beta<(N-k) /(N-$ 1 ), it must be that the expected payoff of applying in mari and that of not applying ((3) and (4), respectively) are indifferent when the buyer's type is $\mu \bar{t}$. That is,

$$
(\mu \bar{t}-\beta \bar{t}) \mathbb{E}_{N-2}^{1-\mu}\left[\mathbf{1}_{j<k-1}+\frac{k-1}{j+1} \mathbf{1}_{j \geq k-1}\right]=\mu \bar{t} \mathbb{E}_{N-2}^{1-\mu}\left[\frac{k-1-j}{N-1-j} \mathbf{1}_{j<k-1}\right]
$$

Applying Lemma 2 in the Appendix, we have

$$
\mu \mathbb{E}_{N-2}^{1-\mu}\left[\frac{k-1-j}{N-1-j} \mathbf{1}_{j<k-1}\right]=\frac{1}{N-1} \mathbb{E}_{N-1}^{1-\mu}\left[(k-1-j) \mathbf{1}_{j<k-1}\right],
$$

and

$$
\begin{aligned}
& \mu \mathbb{E}_{N-2}^{1-\mu}\left[\mathbf{1}_{j<k-1}+\frac{k-1}{j+1} \mathbf{1}_{j \geq k-1}\right] \\
& =\mathbb{E}_{N-2}^{1-\mu}\left[\mathbf{1}_{j<k-1}+\frac{k-1}{j+1} \mathbf{1}_{j \geq k-1}\right]-\frac{1}{N-1} \mathbb{E}_{N-1}^{1-\mu}\left[j \mathbf{1}_{j \leq k-1}+(k-1) \mathbf{1}_{j>k-1}\right] .
\end{aligned}
$$


Substituting these two equations into (7) and rearranging yields

$$
\begin{aligned}
& (1-\beta) \mathbb{E}_{N-2}^{1-\mu}\left[\mathbf{1}_{j<k-1}+\frac{k-1}{j+1} \mathbf{1}_{j \geq k-1}\right] \\
& =\frac{1}{N-1} \mathbb{E}_{N-1}^{1-\mu}\left[(k-1) \mathbf{1}_{j<k-1}+j \mathbf{1}_{j=k-1}+(k-1) \mathbf{1}_{j>k-1}\right] \\
& =\frac{k-1}{N-1} .
\end{aligned}
$$

Thus we have equation (6). As the left-hand side is increasing in $\mu$ by applying Lemma 3 in the Appendix, and

$$
\mathbb{E}_{N-2}^{1}\left[\mathbf{1}_{j<k-1}+\frac{k-1}{j+1} \mathbf{1}_{j \geq k-1}\right]=\frac{k-1}{N-1}, \quad \mathbb{E}_{N-2}^{0}\left[\mathbf{1}_{j<k-1}+\frac{k-1}{j+1} \mathbf{1}_{j \geq k-1}\right]=1 ，
$$

equation (6) has a unique solution for $\mu$ in $(0,1)$ as long as $0<\beta<(N-$ $k) /(N-1)$. For $\beta \geq(N-k) /(N-1), \mu=1$ follows from that the unique solution for (6) is $\mu=1$ when $\beta=(N-k) /(N-1)$.

(Sufficiency). Next we show that the buyer whose type is more than $\mu \bar{t}$ actually participates in mari as long as other buyers are following the equilibrium strategy given in (6). If this buyer does not participate in mari, the expected payoff is (from (4))

$$
\pi_{w}(t)=t \times \mathbb{E}_{N-2}^{1-\mu}\left[\mathbf{1}_{j<k-1}\right]+B_{1}
$$

where $B_{1}$ is the term irrelevant of $t$. If this buyer decides to participate in mari, on the other hand, the expected payoff is (from (3))

$$
\pi_{m}(t)=t \times \mathbb{E}_{N-2}^{1-\mu}\left[\mathbf{1}_{j<k-1}+\frac{k-1}{j+1} \mathbf{1}_{j \geq k-1}\right]+B_{2}
$$


where $B_{2}$ is again the term irrelevant of $t$. Thus, we have that

$$
\frac{\partial}{\partial t} \pi_{m}(t)>\frac{\partial}{\partial t} \pi_{w}(t)
$$

for all $t>\mu \bar{t}$. As the two payoffs are indifferent for type- $\mu \bar{t}$ buyer (that is, $\pi_{m}(\mu \bar{t})=\pi_{w}(\mu \bar{t})$ as shown in the necessity part), we have $\pi_{m}(t)>\pi_{w}(t)$ for all $t>\mu \bar{t}$, and thus we can conclude that the buyer whose type is more than $\mu \bar{t}$ cannot be better off by absenting mari as long as other buyers are following $\mu$.

Finally we show that the buyer whose type $t$ is less than $\mu \bar{t}$ does not participate in mari as long as other buyers are following the symmetric equilibrium strategy $\mu$. For such $t<\mu \bar{t}$, we have

$$
\frac{\partial}{\partial t} \pi_{w}(t)=\mathbb{E}_{N-2}^{1-\mu}\left[x_{N-1-j, k-1-j}\left(\frac{t}{\mu \bar{t}}\right) \times \mathbf{1}_{j<k-1}\right],
$$

from (4). We should note here that $x_{N-1-j, k-1-j}(\cdot)$ is a sort of probability (see (5)), and therefore we have

$$
\frac{\partial}{\partial t} \pi_{w}(t) \leq \mathbb{E}_{N-2}^{1-\mu}\left[\mathbf{1}_{j<k-1}\right]
$$

for all $t<\mu \bar{t}$. As the two payoffs are indifferent for type- $\mu \bar{t}$ buyer (as shown in the necessity part), we can apply similar discussion as in the case of $t>\mu \bar{t}$, and conclude that the buyer whose type is less than $\mu \bar{t}$ cannot be better off by participating in mari as long as other buyers are following $\mu$.

An informal interpretation of the equilibrium is as follows. Without mari, a buyer who failed to win the good in the first round bids higher in the next round than in the first, due to the deterioration of available 
supply relative to current demand. ${ }^{8}$ Hence, the buyer whose value is close to $\bar{t}$ would prefer to win the good in mari with price $\beta \bar{t}$ (although this is higher than the buyer's potential bid in the first round), rather to get into the next round where the bid higher than $\beta \bar{t}$ might be required due to the raise of competitiveness. This is what happens in 2. of the proposition. On the other hand, if $\beta$ is sufficiently high as in 1 . of the proposition so that the bidding higher than $\beta \bar{t}$ would not be needed in the following round after mari even with the raise of competitiveness, then in the equilibrium no buyers participate in mari $(\mu=1)$.

In what follows, we investigate the bidding strategy $\beta$ in the first round in the equilibrium, given the mari participation strategy provided in Proposition 1. As will be shown in Proposition 2, $\beta$ is actually less than $(N-k) /(N-1)$, and therefore participations in mari occur with positive probability in the equilibrium.

In the first-period auction the decision problem facing a buyer is slightly more complex. Again let us take the perspective of one buyer with value $t$ and suppose that all other buyers are following the first-period strategy $\beta$ and mari participation strategy $\mu$.

The equilibrium requires the buyer with type $t$ to bid $\beta t$ in the first stage, but consider what happens if the buyer bids $\beta$ s instead. Then the buyer's payoff is

$$
\Pi(s, t)=\left\{\begin{array}{ll}
s^{N-1}[t-\beta s]+\int_{s}^{z} \pi_{m}(t \mid u) d u^{N-1}+\int_{z}^{1} \pi_{w}(t \mid u) d u^{N-1} & \text { if } s<z \\
s^{N-1}[t-\beta s]+\int_{s}^{1} \pi_{w}(t \mid u) d u^{N-1} & \text { if } s \geq z
\end{array},\right.
$$

\footnotetext{
${ }^{8}$ This effect is one aspect of martingale price path result in the usual sequential descending auction without mari. See Milgrom and Weber [3].
} 
where $z=\min \{t / \mu, 1\}$, and $\pi_{m}(\cdot \mid u)$ and $\pi_{w}(\cdot \mid u)$ are those payoffs given in (3) and (4), respectively, emphasizing that the type of the winner in the first-round, $u$, is included in the function as $\bar{t}=u$. We shall explain the two expressions in due order.

The first term results from the event that the buyer wins the good in the first round. The second term of the first line results from the event that the buyer loses in the first round but participates in mari, and $u$ stands for the highest type among other $N-1$ buyers (that is, the type of the buyer who wins the good in the first round). The third term is the term resulting from the event that the buyer loses the first round, does not participate in mari and then finally wins the good in the auctions after mari. ${ }^{9}$

It is possible, however, that the buyer might bid sufficiently large price in the first round so that when the buyer happens to lose in the first round even with this high bid, it never becomes optimal for him to participate in mari, as the type of the winner in the first round is supposed to be extremely high. The second line in the expression (9) corresponds to such biddings. The first term results from the event that the buyer wins the good in the first round, and the second term is the event that the buyer loses the first round, does not (definitely) participate in mari and then finally wins the good in the auctions after mari.

In the symmetric equilibrium, the buyer with type $t$ bids $\beta t$ in the first round and therefore the payoff is calculated according to the first line in (9). An increase in the bid of this buyer will increase the probability of winning the good, but at the same time this will reduce the gain from the winning in the first round (first term) as well as the gain from the event that the

\footnotetext{
${ }^{9}$ The buyer with sufficiently large type $(t>\mu)$ always finds it optimal to participate in mari whatsoever the first round price $\beta \bar{t}$ could be. For such buyers, the expected payoff $\Pi(s, t)$ is calculated with $z=1$, and the third term vanishes.
} 
buyer loses the first round but wins the good in mari (second term). To get some idea about how these effects balance off, we begin with a heuristic derivation of symmetric equilibrium strategies.

Differentiating $\Pi(s, t)$ with respect to $s$ provides us with

$$
\begin{aligned}
\frac{\partial}{\partial s} \Pi(s, t) & =(N-1) s^{N-2}(t-\beta s)-\beta s^{N-1}-\pi_{m}(t \mid s)(N-1) s^{N-2} \\
& =s^{N-2}\left\{(N-1)\left[(t-\beta s)-\pi_{m}(t \mid s)\right]-\beta s\right\}
\end{aligned}
$$

At a symmetric equilibrium, the first-order condition should be satisfied in substituting $s=t$. That is, we have

$$
(N-1)\left\{(t-\beta t)-\pi_{m}(t \mid t)\right\}=\beta t \quad \text { for all } t
$$

The left-hand side corresponds to the marginal benefit by an increment of $s$, whereas the right-hand side corresponds to the marginal cost (the marginal increment in payment), and the two effects are balanced off at the point $s=t$. Substituting $\pi_{m}(t \mid t)$ defined in (3), we have

$$
(N-1)\left\{(1-\beta)-(1-\beta) \mathbb{E}_{N-2}^{1-\mu}\left[\mathbf{1}_{j<k-1}+\frac{k-1}{j+1} \mathbf{1}_{j \geq k-1}\right]\right\}=\beta,
$$

and by substituting (6), we have $\beta=(N-k) / N$.

The derivation of $\beta$ is only heuristic because (11) is merely a necessary condition that will be satisfied in the equilibrium. The next proposition verifies that this constitutes the equilibrium and provides the full characterization of $\mu$.

Proposition 2. Symmetric equilibrium strategies in the sequential descending auction with mari are given by the bidding strategy in the first round $\beta=(N-k) / N$ 
and mari participation strategy $\mu$ given as the unique solution of

$$
\mathbb{E}_{N-2}^{1-\mu}\left[\mathbf{1}_{j<k-1}+\frac{k-1}{j+1} \mathbf{1}_{j \geq k-1}\right]=\frac{k-1}{N-1} \frac{N}{k}
$$

Proof. Suppose that the other $N-1$ buyers follow the strategy $\beta=(N-k) / N$. Consider a buyer with type $t$ who is going to bid an amount $\beta$ s instead of $\beta t$. We are to show that

$$
\Pi(t, t)>\Pi(s, t) \quad \text { for all } s \neq t
$$

by showing

$$
\begin{array}{ll}
\frac{\partial}{\partial s} \Pi(s, t)>0 & \text { for all } s<t \\
\frac{\partial}{\partial s} \Pi(s, t)<0 & \text { for all } s>t
\end{array}
$$

For $s<t$, we have from (9) that

$$
\begin{aligned}
\frac{\partial}{\partial s} \Pi(s, t) & =(N-1) s^{N-2}(t-\beta s)-\beta s^{N-1}-\pi_{m}(t \mid s)(N-1) s^{N-2} \\
& =s^{N-2}\left\{(N-1)\left[(t-\beta s)-\pi_{m}(t \mid s)\right]-\beta s\right\}
\end{aligned}
$$

As long as the other buyers are following the strategy $\beta,(10)$ is satisfied for all $t$, and in particular, $\beta s=(N-1)\left\{(s-\beta s)-\pi_{m}(s \mid s)\right\}$. Therefore,

$$
\frac{\partial}{\partial s} \Pi(s, t)=(N-1) s^{N-2}\left\{f_{s}(t)-f_{s}(s)\right\}
$$

where we defined $f_{s}(t)$ as $f_{s}(t)=t-\beta s-\pi_{m}(t \mid s)$. It is easy to see that

$$
f_{s}^{\prime}(t)=1-\frac{\partial}{\partial t} \pi_{m}(t \mid s)
$$




$$
=1-\mathbb{E}_{N-2}^{1-\mu}\left[\mathbf{1}_{j<k-1}+\frac{k-1}{j+1} \mathbf{1}_{j \geq k-1}\right]>0 .
$$

Thus we can conclude that $\partial \Pi(s, t) / \partial s>0$ for all $s<t$.

For $t<s \leq z$, the partial derivative is the same form as in (13), and we have $\partial \Pi(s, t) / \partial s<0$ for all $t<s \leq z$ by (14).

For $s>z$, we can prove the result in a similar manner. We have the derivative

$$
\frac{\partial}{\partial s} \Pi(s, t)=(N-1) s^{N-2}\left\{g_{s}(t)-g_{s}(s)\right\},
$$

where $g_{s}(t)=t-\beta s-\pi_{w}(t \mid s)$. As $t / \mu<s$ in this case, $\pi_{w}(t \mid s)$ corresponds to the second line of the definition in (4), and therefore

$$
\frac{\partial}{\partial t} \pi_{w}(t \mid s) \leq \mathbb{E}_{N-2}^{1-\mu}\left[\mathbf{1}_{j<k-1}\right]<1
$$

from (8). Thus we have $g_{s}^{\prime}(t)>0$ for all $t$, and $\partial \Pi(s, t) / \partial s<0$ for all $s>z$.

We have established that

$$
\Pi(t, t)>\Pi(s, t) \quad \text { for all } s \neq t
$$

that is, the type- $t$ buyer finds it optimal to bid $\beta t$ as long as other buyers are following the strategy $\beta$.

Substituting $\beta=(N-k) / N$ into (6) yields the equation (12).

\section{Efficiency vs. Economy of Time}

In this section, we argue that mari extensively speeds up the market procedure at the cost of sufficiently small loss of efficiency.

As shown in Proposition 2, in the symmetric equilibrium, participation 
in mari occurs with strictly positive probability. The possibility of random allocation in mari causes ex ante inefficiency, whereas selling several goods at one time in mari may contribute to the economy of time at the market. To formally evaluate the degree of inefficiency as well as the level of economy of time, we define two measures, the "efficiency loss rate" ( $L R$, hereafter) and the "round reduction rate" ( $R R$, hereafter). Formal (mathematical) definitions of those two concepts will be given in the proof of Theorem with some relevant notions to smoothen the outset of the proof. In the following, we present casual definitions of the concepts and argue how the trade-off of our interest can be investigated mathematically.

We refer to $L R$ as the ratio of the expected efficiency loss in mari to the expected surplus when the goods are allocated efficiently between the participants. Thus, $L R=0$ says that the goods will be allocated perfectly efficiently between buyers and hence there will be no loss of surplus in mari (i.e., first best allocation), whereas $L R=1$ would describe the situation where the latent surplus from the auction is totally vanished and the resulting surplus is going to be zero.

On the other hand, $R R$ is defined as the ratio of the expected number of the goods sold in mari to $k-1$ (the number of remaining goods in the beginning of mari). Thus, $R R=1$ says that all of the remaining $k-1$ goods will be "cleaned up" at one time in mari, whereas $R R=0$ refers to the case where none of the remaining goods will be sold and mari has no contribution to the economy of time in the auction process.

Figure 1 plots the values of $L R$ and $R R$ for various sets of parameters $2 \leq k \leq 10$ and $k+1 \leq n \leq 5 k$. The point on the upper-left corner is for the case of $k=2$ and $n=3$, while the one on the lower-right corner is for the case in which $k=10$ and $n=11$. The figure shows that for most 


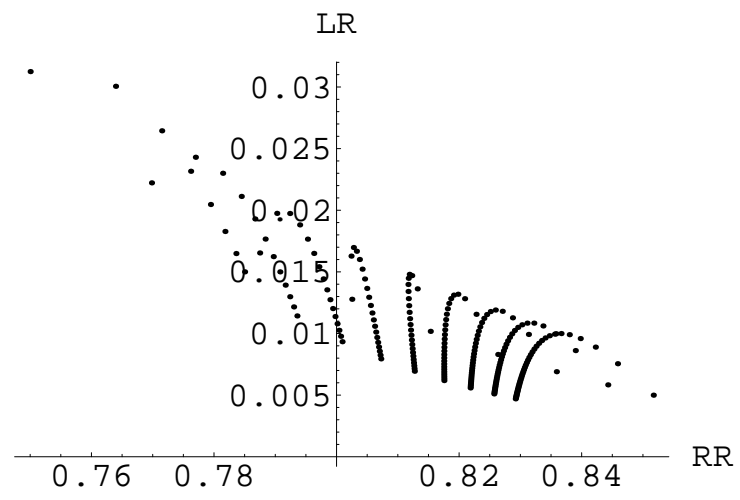

Figure 1: $L R$ and $R R$

of the parameters, the $L R$ tends to be small with relatively large values of $R R$. Put in another way, the figure indicates that mari extensively speeds up the market procedure (high $R R$ ) at the cost of sufficiently small loss of efficiency (low $L R$ ). For instance, van den Berg et al. [2] reports that $k$ is 5.98 on average at the Aalsmeer Flower Auction in the Netherlands. The figure shows for $k=6$ that we have more than $80 \%$ of $R R$ in exchange for less than $1.5 \%$ of $L R$.

To formally investigate the tradeoff between $L R$ and $R R$, we have the following convergence theorem.

Theorem. In the linear symmetric equilibrium of the sequential descending auction with mari, we have

$$
L R \rightarrow 0 \text { and } R R \rightarrow 1 \quad \text { as } \quad N, k \rightarrow \infty \text { with } \frac{k}{N}=\alpha
$$

Prooffor $L R \rightarrow 0$. Let $W_{N, k}^{*}$ denote the expected surplus when the $k-1$ items are allocated efficiently between $N-1$ buyers $^{10}$, and $W_{N, k}^{m}$ denote the

\footnotetext{
${ }^{10}$ In the beginning of the mari period, one unit out of $k$ items is already sold to one buyer out of $N$ buyers, and therefore we are interested in how the remaining $k-1$ items are to be allocated between the remaining $N-1$ buyers as far as we are concerned with
} 
expected surplus attained under the mari auction discussed in the paper. Then the rate $L R$ can be written as $\left(W_{N, k}^{*}-W_{N, k}^{m}\right) / W_{N, k}^{*}$. In what follows, we are to prove $L R \rightarrow 0$ by showing $1-L R=W_{N, k}^{m} / W_{N, k}^{*} \rightarrow 1$ in $N, k \rightarrow \infty$.

In accordance with the definition of $W_{N, k}^{*}$, we let $W_{N, k}^{*}(j)$ denote the expected surplus when $j$ buyers participate in mari and $k-1$ goods are allocated efficiently between them. In a similar way, we let $W_{N, k}^{m}(j)$ denote the expected surplus when $j$ buyers participate in mari and $k-1$ goods are allocated under the mari rule. Note that we have

$$
W_{N, k}^{*}=E_{N-1}^{1-\mu_{N, k}}\left[W_{N, k}^{*}(j)\right] \quad \text { and } \quad W_{N, k}^{m}=E_{N-1}^{1-\mu_{N, k}}\left[W_{N, k}^{m}(j)\right]
$$

where $\mu_{N, k}$ is the mari-participation threshold strategy in the equilibrium with $N$ buyers and $k$ items (see Propositions 1 and 2; in what follows we omit the subscripts $N$ and $k$ ).

Our main interest is on $j \geq k-1$, in which case the resulting allocation would be different between efficient allocation and mari. Suppose that, in the first round, some buyer with value $\bar{t}$ has pushed the button and won the good. Now the values of remaining buyers are distributed on $U[0, \bar{t}]$, and those buyers with values in $[\mu \bar{t}, \bar{t}]$ would participate in mari in the equilibrium. Then for $j \geq k-1$, we have

$$
\begin{aligned}
& W_{N, k}^{*}(j)=\bar{t}(k-1)\left\{(1-\mu)\left(1-\frac{k}{2(j+1)}\right)+\mu\right\}, \\
& W_{N, k}^{m}(j)=\bar{t}(k-1) \frac{1+\mu}{2} .
\end{aligned}
$$

The first equation is the expected surplus when $k-1$ goods are allocated the relative efficiency of mari auction. 
efficiently to the top $k-1$ buyers out of $j$ participants ${ }^{11}$, and the second equation is the expected surplus when $k-1$ goods are allocated randomly between $j$ participants, each of whose type is distributed on $U[\mu \bar{t}, \bar{t}]$ with the expected value of $\bar{t}(\mu+1) / 2$.

Firstly, we have

$$
\frac{W_{N, k}^{m}}{W_{N, k}^{*}} \geq \frac{w_{N, k}^{m}}{W_{N, k}^{*}}
$$

as a lower bound for $1-L R$, where $w_{N, k}^{m}$ denotes the expected surplus generated by the event $j \leq(1+\epsilon)(k-1)$ in the mari auction for a given small number $\epsilon>0$. In what follows, we decompose this lower bound as

$$
\frac{w_{N, k}^{m}}{W_{N, k}^{*}}=\frac{w_{N, k}^{*}}{W_{N, k}^{*}} \times \frac{w_{N, k}^{m}}{w_{N, k}^{*}}
$$

where $w_{N, k}^{*}$ is similarly denoted as the expected surplus generated by the event $j \leq(1+\epsilon)(k-1)$ when the goods are allocated efficiently between $j$ participants, and investigate each term's further lower bound and its asymptotic behavior.

For the relationship between $w_{N, k}^{*}$ and $W_{N, k^{\prime}}^{*}$ we have

$$
W_{N, k}^{*}=w_{N, k}^{*}+E_{N-1}^{1-\mu}\left[W_{N, k}^{*}(j) \mathbf{1}_{j>(1+\epsilon)(k-1)}\right]
$$

\footnotetext{
${ }^{11}$ Note that the expectation of $i$ th order statistic in $j$ draws from the uniform distribution $U[\mu \bar{t}, \bar{t}]$ is $\mu \bar{t}+(\bar{t}-\mu \bar{t})(j-i+1) /(j+1)$, and therefore we have

$$
W_{N, k}^{*}(j)=\sum_{i=0}^{k-1}\left\{\mu \bar{t}+(\bar{t}-\mu \bar{t}) \frac{j-i+1}{j+1}\right\}=\bar{t}(k-1)\left\{(1-\mu)\left(1-\frac{k}{2(j+1)}\right)+\mu\right\} .
$$

Note also that the (unconditional) expected surplus if allocated efficiently is calculated as

$$
W_{N, k}^{*}=\bar{t}(k-1)\left(1-\frac{k}{2 N}\right) .
$$
}


by the definition of $w_{N, k}^{*}$. For $(1+\epsilon)(k-1)<j \leq N-1$, it is clear that $W_{N, k}^{*}(j)$ is at most

$$
\bar{t}(k-1)\left\{(1-\mu)\left(1-\frac{k}{2 N}\right)+\mu\right\},
$$

and therefore a lower bound of $w_{N, k}^{*} / W_{N, k}^{*}$ is given by

$$
\frac{w_{N, k}^{*}}{W_{N, k}^{*}} \geq 1-\frac{(1-\mu)\left(1-\frac{k}{2 N}\right)+\mu}{1-\frac{k}{2 N}} E_{N-1}^{1-\mu}\left[\mathbf{1}_{j>(1+\epsilon)(k-1)}\right],
$$

where the denominator in the second term comes from the fact that $W_{N, k}^{*}=$ $\bar{t}(k-1)(1-k /(2 N))$ (see footnote 11$)$.

For $w_{N, k}^{m} / w_{N, k}^{*}$ part in (16), we focus our attention to the event that $j \leq(1+\epsilon)(k-1)$. It is clear that

$$
W_{N, k}^{*}(j) \leq \bar{t}(k-1)\left\{(1-\mu)\left(1-\frac{k}{2((1+\epsilon)(k-1)+1)}\right)+\mu\right\}
$$

for each $j \leq(1+\epsilon)(k-1)$, and therefore a lower bound for $w_{N, k}^{m} / w_{N, k}^{*}$ is given by

$$
\begin{aligned}
\frac{w_{N, k}^{m}}{w_{N, k}^{*}} & \geq \frac{\bar{t}(k-1) \frac{1+\mu}{2}}{\bar{t}(k-1)\left\{(1-\mu)\left(1-\frac{k}{2(1+\epsilon)(k-1)+1)}\right)+\mu\right\}} \\
& =1 /\left(1+\frac{1-\mu}{1+\mu} \frac{\epsilon(k-1)}{(1+\epsilon)(k-1)+1}\right) \\
& \geq 1 /\left(1+\frac{k / N}{2-k / N} \frac{\epsilon(k-1)}{(1+\epsilon)(k-1)+1}\right),
\end{aligned}
$$

where the last inequality comes from the condition that $\mu \geq \beta=(N-k) / N$ in the equilibrium. 
From (15), (16), (17), and (18), we have

$$
\begin{aligned}
1-L R & \equiv \frac{W_{N, k}^{m}}{W_{N, k}^{*}} \\
& \geq \frac{1-\frac{(1-\mu)\left(1-\frac{k}{2 N}\right)+\mu}{1-\frac{k}{2 N}} E_{N-1}^{1-\mu}\left[\mathbf{1}_{j>(1+\epsilon)(k-1)}\right]}{1+\frac{k / N}{2-k / N} \frac{\epsilon(k-1)}{(1+\epsilon)(k-1)+1}},
\end{aligned}
$$

and by applying Lemma 4 in the Appendix,

$$
\liminf _{N \rightarrow \infty}(1-L R) \geq \frac{1}{1+\frac{\alpha}{2-\alpha} \frac{\epsilon}{1+\epsilon}}
$$

As $\epsilon>0$ can be arbitrarily small, this completes the proof.

Proof for $R R \rightarrow 1$. Our proof proceeds as follows: 1 . we establish that $\liminf _{N \rightarrow \infty}(1-\mu)(N-1) /(k-1) \geq 1$, and 2 . we show that $R R \rightarrow 1$ under the condition derived in step 1. by applying Arratia-Gordon [1] method as in the proof for $L R \rightarrow 0$. (that is, the expected number of buyers participating in mari in the equilibrium, $(1-\mu)(N-1)$, converges to something slightly greater than the number of remaining items, $k-1)$.

1. Firstly we should note that the left-hand side of the equilibrium condition (12) represents the probability that "a buyer wins the item in mari if the buyer participates in $i^{\prime \prime}$ in the equilibrium. Rearranging the equation in terms of the probability that "a buyer loses in mari if the buyer participates in it", we have

$$
1-\mathbb{E}_{N-2}^{1-\mu}\left[\mathbf{1}_{j<k-1}+\frac{k-1}{j+1} \mathbf{1}_{j \geq k-1}\right]=1-\frac{k-1}{N-1} \frac{N}{k}=\frac{1-\alpha}{\alpha} \frac{1}{N-1}
$$

where $\alpha=k / N$. As the event that "a buyer loses in mari if the buyer 
participates in it" is included in the event that "the number of other buyers participating in mari is larger than $k-1 "$,

$$
\mathbb{E}_{N-2}^{1-\mu}\left[\mathbf{1}_{j \geq k-1}\right] \geq 1-\mathbb{E}_{N-2}^{1-\mu}\left[\mathbf{1}_{j<k-1}+\frac{k-1}{j+1} \mathbf{1}_{j \geq k-1}\right] .
$$

Thus we have

$$
\mathbb{E}_{N-2}^{1-\mu}\left[\mathbf{1}_{j \geq k-1}\right] \geq \frac{1-\alpha}{\alpha} \frac{1}{N-1} .
$$

Given the inequality (19), we can show $\lim _{N \rightarrow \infty}(1-\mu)(N-2) /(k-1) \geq 1$ by contradiction. Specifically, we are to show that "if we suppose that $\lim _{N \rightarrow \infty}(1-\mu)(N-2) /(k-1)<1$, then it contradicts the inequality (19)".

Suppose that we have $\lim _{N \rightarrow \infty}(1-\mu)(N-2) /(k-1)<1$. Then we can apply Lemma 1 (Arratia-Gordon Theorem) by seeing as $p:=1-\mu$ and $\alpha:=(k-1) /(N-2)$, and would have

$$
\mathbb{E}_{N-2}^{1-\mu}\left[\mathbf{1}_{j \geq k-1}\right] \leq e^{-(N-2) \cdot H}
$$

This inequality would say that $\mathbb{E}_{N-2}^{1-\mu}\left[\mathbf{1}_{j \geq k-1}\right]$ is the order of $1 / e^{N}$, which contradicts the inequality (19) stating that the left-hand side has no less order than $1 / N$. Thus we have

$$
\lim _{N \rightarrow \infty} \frac{(1-\mu)(N-2)}{k-1} \geq 1
$$

2. $R R$ can be written as follows:

$$
R R=\frac{\mathbb{E}_{N-1}^{1-\mu}\left[j \mathbf{1}_{j<k-1}\right]+\mathbb{E}_{N-1}^{1-\mu}\left[(k-1) \mathbf{1}_{j \geq k-1}\right]}{k-1} .
$$

The first term in the numerator is regarding the event that $j<k-1$ in which case the number of items sold in mari is only $j$, whereas the second 
term in the numerator is regarding the event that $j \geq k-1$ in which case the number of items sold in mari is $k-1$, and the sum of these two terms is divided by the number of remaining goods, $k-1$. We are to show $R R \rightarrow 1$ by firstly giving a lower bound for $R R$ and then showing that the lower bound converges to 1 .

For any arbitrarily given number $\epsilon>0$, we have

$$
\begin{aligned}
R R & \geq \frac{\mathbb{E}_{N-1}^{1-\mu}\left[0 \cdot \mathbf{1}_{j<(1-\epsilon)(k-1)}+(1-\epsilon)(k-1) \cdot \mathbf{1}_{j \geq(1-\epsilon)(k-1)}\right]}{k-1}, \\
& =(1-\epsilon) \mathbb{E}_{N-1}^{1-\mu}\left[\mathbf{1}_{j \geq(1-\epsilon)(k-1)}\right] \\
& \geq(1-\epsilon) \mathbb{E}_{N-2}^{1-\mu}\left[\mathbf{1}_{j \geq(1-\epsilon)(k-1)}\right] \\
& =(1-\epsilon)\left\{1-\mathbb{E}_{N-2}^{1-\mu}\left[\mathbf{1}_{j<(1-\epsilon)(k-1)}\right]\right\} .
\end{aligned}
$$

As we have $\lim _{N \rightarrow \infty}(1-\mu)(N-2) /(k-1) \geq 1$ established in step 1., we can apply Lemma 1 (Arratia-Gordon Theorem) and have ${ }^{12}$

$$
\mathbb{E}_{N-2}^{1-\mu}\left[\mathbf{1}_{j<(1-\epsilon)(k-1)}\right] \leq e^{-(N-2) \cdot H} \rightarrow 0 \quad \text { as } N \rightarrow \infty
$$

As $\epsilon>0$ can be arbitrarily small, this completes the proof.

\section{Summary}

In this paper we introduced the model that formulates sequential descending auction with mari, and showed that it speeds up the market procedure at the cost of sufficiently small loss of efficiency.

Such mari rules are used in reality at various wholesale flower mar-

\footnotetext{
${ }^{12}$ From the symmetricity of binomial distributions, we can construct the "mirror" of Arratia-Gordon Theorem as follows: If $0<\alpha<p<1$, then we have $\mathbb{E}_{N}^{p}\left[\mathbf{1}_{j \leq \alpha N}\right] \leq e^{-N \cdot H(\alpha, p)}$.
} 
kets in Japan. We believe that the result in the paper provides a partial justification for the introduction of the rule in other markets.

\section{Appendix}

Lemma 1 (Arratia-Gordon). If $0<p<\alpha<1$, then we have

$$
\mathbb{E}_{N}^{p}\left[\mathbf{1}_{j \geq \alpha N}\right] \leq \exp \{-N \cdot H(\alpha, p)\},
$$

where $H(\alpha, p)$ is the Kullback-Liebler distance satisfying

$$
H(\alpha, p) \equiv \alpha \ln \frac{\alpha}{p}+(1-\alpha) \ln \frac{1-\alpha}{1-p}
$$

Proof. See Theorem 1 in Arratia and Gordon [1].

Lemma 2. For $\mathbb{E}_{m}^{1-\mu}[f(j)] \equiv \sum_{i=0}^{m}\left(\begin{array}{c}m \\ i\end{array}\right)(1-\mu)^{i} \mu^{m-i} f(i)$, we have

$$
\begin{aligned}
\mu \mathbb{E}_{m}^{1-\mu}[f(j)] & =\frac{1}{m+1} \mathbb{E}_{m+1}^{1-\mu}[(m-j+1) f(j)], \\
(1-\mu) \mathbb{E}_{m}^{1-\mu}[f(j)] & =\frac{1}{m+1} \mathbb{E}_{m+1}^{1-\mu}[j f(j-1)] .
\end{aligned}
$$

Proof.

$$
\begin{aligned}
\mu \mathbb{E}_{m}^{1-\mu}[f(j)] & =\sum_{i=0}^{m}\left(\begin{array}{c}
m \\
i
\end{array}\right)(1-\mu)^{i} \mu^{m-i+1} f(i) \\
& =\sum_{i=0}^{m}\left(\begin{array}{c}
m+1 \\
i
\end{array}\right) \frac{m-i+1}{m+1}(1-\mu)^{i} \mu^{m-i+1} f(i) \\
& =\sum_{i=0}^{m+1}\left(\begin{array}{c}
m+1 \\
i
\end{array}\right) \frac{m-i+1}{m+1}(1-\mu)^{i} \mu^{m-i+1} f(i) \\
& =\frac{1}{m+1} \mathbb{E}_{m+1}^{1-\mu}[(m-j+1) f(j)] .
\end{aligned}
$$


Similarly,

$$
\begin{aligned}
(1-\mu) \mathbb{E}_{m}^{1-\mu}[f(j)] & =\sum_{i=0}^{m}\left(\begin{array}{c}
m \\
i
\end{array}\right)(1-\mu)^{i+1} \mu^{m-i} f(i) \\
& =\sum_{i=0}^{m}\left(\begin{array}{c}
m+1 \\
i+1
\end{array}\right) \frac{i+1}{m+1}(1-\mu)^{i+1} \mu^{m-i} f(i) \\
& =\sum_{i=1}^{m+1}\left(\begin{array}{c}
m+1 \\
i
\end{array}\right) \frac{i}{m+1}(1-\mu)^{i} \mu^{m+1-i} f(i-1) \\
& =\frac{1}{m+1} \mathbb{E}_{m+1}^{1-\mu}[j f(j)] .
\end{aligned}
$$

Lemma 3. If $f(j) \geq f(j+1)$ for all $j$ and $f(j)>f(j+1)$ for some $j$, then $\mathbb{E}_{m}^{1-\mu}[f(j)]$ is (strictly) increasing in $\mu$.

Proof.

$$
\begin{aligned}
\frac{\partial}{\partial \mu} \mathbb{E}_{m}^{1-\mu}[f(j)]= & \sum_{i=1}^{m-1}\left(\begin{array}{c}
m \\
i
\end{array}\right)\left\{-(1-\mu)^{i-1} \mu^{m-i}+(m-i)(1-\mu)^{i} \mu^{m-i-1}\right\} f(i) \\
& +m \mu^{m-1} f(0)-(1-\mu)^{m-1} f(m) \\
= & \sum_{i=0}^{m-1}\left\{\left(\begin{array}{c}
m \\
i
\end{array}\right)(m-i) f(i)-\left(\begin{array}{c}
m \\
i+1
\end{array}\right)(i+1) f(i+1)\right\}(1-\mu)^{i} \mu^{m-i-1} \\
= & \sum_{i=0}^{m-1} \frac{m !}{i !(m-i-1) !}(1-\mu)^{i} \mu^{m-i-1}[f(i)-f(i+1)] \\
> & 0 .
\end{aligned}
$$

Lemma 4. If $N$ is large enough to satisfy $k / N<(1+\epsilon)(k-1) /(N-1)$ for a given $\epsilon>0$, then

$$
E_{N-1}^{1-\mu}\left[\mathbf{1}_{j>(1+\epsilon)(k-1)}\right] \rightarrow 0 \quad \text { as } N \rightarrow \infty
$$


Proof. In the equilibrium it should be that $1-\mu \leq 1-\beta=k / N$, and therefore we have

$$
E_{N-1}^{1-\mu}\left[\mathbf{1}_{j>(1+e)(k-1)}\right] \leq E_{N-1}^{k / N}\left[\mathbf{1}_{j>(1+\epsilon)(k-1)}\right] .
$$

If $k / N<(1+\epsilon)(k-1) /(N-1)$, then Lemma 1 can be applied, which provides us with

$$
E_{N-1}^{k / N}\left[\mathbf{1}_{j>(1+\epsilon)(k-1)}\right] \leq e^{-(N-1) \cdot H\left(\frac{(1+e)(k-1)}{N-1}, \frac{k}{N}\right),}
$$

where $H$ is the Kullback-Liebler distance converging to a finite value as $N \rightarrow \infty$.

\section{References}

[1] Arratia, R., and L. Gordon (1989), “Tutorial on Large Deviations for the Binomial Distribution", Bulletin of Mathematical Biology 51, 125-131.

[2] van den Berg, G. J., J. C. van Ours, and M. P. Pradhan (2001), “The Declining Price Anomaly in Dutch Rose Auctions", American Economic Review 91, 1055-62.

[3] Milgrom, P., and R. Weber (2000), "A Theory of Auctions and Competitive Bidding, II", in P. Klemperer ed., The Economic Theory of Auctions, Edward Elgar.

[4] Weber, R. J. (1983), “Multiple-Object Auctions", in R. EngelbrechtWiggans ed., Auctions, Bidding, and Contracting: Uses and Theory, New York University Press. 